Volume 00, Number 0, Pages 000-000

$\mathrm{S}$ (XX)0000-0

\title{
CONTROL LYAPUNOV FUNCTIONS AND ZUBOV'S METHOD
}

\author{
FABIO CAMILLI, LARS GRÜNE, AND FABIAN WIRTH
}

\begin{abstract}
For finite dimensional nonlinear control systems we study the relation between asymptotic null-controllability and control Lyapunov functions. It is shown that control Lyapunov functions may be constructed on the domain of asymptotic null-controllability as viscosity solutions of a first order PDE that generalizes Zubov's equation. The solution is also given as the value function of an optimal control problem from which several regularity results may be obtained.
\end{abstract}

\section{INTRODUCTION}

A fundamental question in the qualitative theory of dynamical systems concerns the stability of fixed points or more generally attractors. In his seminal thesis Lyapunov showed that a sufficient stability condition can be obtained in terms of a positive definite function that decreases along the trajectories of the system, or as we say today the existence of a Lyapunov function implies asymptotic stability. Converse theorems state that certain stability properties imply the existence of a Lyapunov function. Early results in this direction were obtained by Persidskii, see the discussion in [19, Chapter VI], Massera [24] and Kurzweil [20]. However, in recent times these results have been extended in several directions to cover perturbed systems and differential inclusions $[22,11,35]$.

While for linear systems a constructive procedure to find Lyapunov functions has already been given by Lyapunov, the first general constructive procedure to find Lyapunov functions was obtained by Zubov [37]. Namely, a Lyapunov function on the domain of attraction of an asymptotically stable fixed point $x^{*} \in \mathbb{R}^{n}$ of the system

$$
\dot{x}(t)=f(x(t)), \quad t \in \mathbb{R}, x \in \mathbb{R}^{n}
$$

may be found by solving the 1st order PDE, called Zubov's equation,

$$
D v(x) f(x)=-h(x)(1-v(x)) \sqrt{1+\|f\|^{2}} \quad x \in \mathbb{R}^{n},
$$

under the condition that $v(0)=0$. Here $h$ is an auxiliary function, see $[37,19]$ for details. This method has been recently extended by the authors to the case of perturbed systems, see [8] where also a discussion of the impact of Zubov's result may be found. Further constructive approaches valid for $C^{2}$ systems and based on approximations by radial basis functions, respectively on a linear programming approach have recently been described in $[17,18]$.

In this paper we investigate the question of existence of Lyapunov functions for systems with inputs or control systems. While for (perturbed) ordinary differential

Received by the editors 06.05.2004.

Supported by Science Foundation Ireland under grant 00/PI.1/C067. 
equations the property of interest is stability, for systems with control inputs a basic question concerns the existence of control functions steering the system to a desired target. Consider a control system

$$
\dot{x}(t)=f(x(t), u(t)),
$$

where $x \in \mathbb{R}^{n}$ denotes the state, $u \in \mathbb{R}^{m}$ denotes the input, and where $f$ is sufficiently regular with $f(0,0)=0$. We call a point $x_{0} \in \mathbb{R}^{n}$ asymptotically controllable to 0 if there exists a measurable, essentially bounded function $u_{0}: \mathbb{R}_{+} \rightarrow \mathbb{R}^{m}$ such that the corresponding solution $\varphi\left(t, x_{0}, u_{0}\right)$ of $(1.1)$ satisfies $\varphi\left(t, x_{0}, u_{0}\right) \rightarrow 0$ for $t \rightarrow \infty$. The domain of asymptotic null-controllability is the collection of all points that are asymptotically controllable to 0 .

Also for this concept there is a Lyapunov theory which has received widespread attention in recent years. In contrast to the case of ordinary differential equations, where smooth Lyapunov functions always exist for asymptotically stable systems, it is not reasonable to require too many regularity properties of Lyapunov functions for controllability questions. For this reason it is now standard to formulate the concept of a control Lyapunov function in nondifferential terms. To formulate this recall that a function $V: \mathbb{R}^{n} \rightarrow \mathbb{R}$ is called positive definite, if $V(x) \geq 0$ for all $x \in \mathbb{R}^{n}$ and $V(x)=0$ iff $x=0$. The function $V$ is proper if preimages of compact sets are compact. A positive definite, proper function $V$ is called a control-Lyapunov function (CLF) for (1.1) if there is a positive definite function $W$ such that for every compact set $X \subset \mathbb{R}^{n}$ there is a compact set $U_{X}$ of control values so that $V$ is a continuous viscosity supersolution of

$$
\max _{u \in U_{X}}-D V(x) f(x, u) \geq W(x), \quad x \in X .
$$

For the definition of viscosity solutions we refer to [4]. In many articles control Lyapunov functions are defined in terms of proximal subgradients of $V$, but the two notions are in fact equivalent, [9].

While design techniques using Lyapunov functions have been popular in applied control theory for a long time, the systematic study of control Lyapunov functions only started with Artstein [1], who proved for the case of systems affine in the control term $u$ that the existence of a smooth CLF is equivalent to stabilizability by continuous state feedback. For general systems of the form (1.1) the existence of a global continuous CLF is equivalent to global asymptotic null controllability [29]. Interestingly, the existence of a differentiable CLF is equivalent to the existence of (discontinuous) stabilizing feedbacks that are robust with respect to measurements in the state, [21]. Now in general asymptotic nullcontrollability does not imply the existence of continuous stabilizing feedback as there may be topological obstructions to this which even carry over to the case of upper semicontinuous set-valued feedbacks, [7, 12, 27]. For this reason discontinuous feedbacks and associated solution concepts have been one of the focal points of the research on CLF's in recent times starting with [10]. In this context it has been shown by Clarke et al. [9], Rifford $[25,26]$ using tools from nonsmooth analysis that semiconcavity of the CLF is an essential tool in order to establish the existence of feedback with nice properties.

Usually, the knowledge of a CLF requests a certain structure of the control system, while a general procedure for its determination is not available. Constructive approaches have therefore received widespread attention in literature, most notably with techniques known as backstepping and forwarding [16, 28], which however, rely heavily on the differentiability of the CLF that is obtained. In this article we aim to 
derive a constructive approach by going back to the original ideas for the construction of control Lyapunov functions. Here constructive is to be understood in the way that we determine a class of PDEs which have unique solutions in the viscosity sense that are control Lyapunov functions.

It is a classical approach to the problem to regard CLFs as solutions of a steady state Hamilton-Jacobi (HJ) equation. In the uncontrolled case this may be regarded as one of the central elements of the work of Zubov [19]. In [15] the connection between smooth CLFs and HJ equations has been studied in detail. In particular, it is shown in that paper that smooth CLFs may always be interpreted as value functions of an appropriate optimal control problem. This "inverse optimality" property can be exploited in several ways [16]. In a different approach, in [14] a CLF was obtained by truncating series expansion of analytical solutions of HJ equations in an approach very similar to original studies around Zubov's equation.

In the present paper we use ideas from [8] where, for the case of a perturbed system, the classical Zubov method was reinterpreted using a suitable notion of weak solution. For controlled or perturbed systems Zubov's equation becomes a nonlinear $1^{\text {st }}$ order PDE of Hamilton-Jacobi type and it is well known that this class of equations does not admit, in general, classical solutions. Therefore a suitable concept of weak solution has to be introduced and the one of viscosity solution seems to be appropriate, see [8], [23].

We use this generalization of Zubov's method to construct a CLF for a finite dimensional nonlinear control system, that is asymptotically null controllable in a neighborhood of the origin. Our aim is to determine a CLF as (i) an optimal value function of a suitable control problem and (ii) as unique viscosity solution to a suitable HJ equations which is a generalization of the Zubov's equation.

Concerning the first point, i.e. the connection between CLF and optimal control problems, our procedure can be viewed as an extension of [29] where the equivalence between asymptotic null controllability and the existence of a CLF has been proved using an optimal control approach. The significant advantage of the characterization of a CLF as unique viscosity solution of the generalized Zubov equation is that it can used as the basis for its numerical approximation.

From the point of view of the PDE approach the equation presents some difficulties when attacked using the standard theory of viscosity solution because of the unbounded control set, see [5], [13], [34], [33] for related papers. In the proof of the necessary comparison result we use the local asymptotic controllability to obtain a local comparison result in a neighborhood of the origin. We then extend the comparison result to all $\mathbb{R}^{n}$ taking advantage, as in the classical Zubov method, of the freedom in the choice of cost function of the associated control problem. For this reason we can make rather general assumptions on the dependence of the dynamics respect to the control variable compensating them with an appropriate choice of the cost.

We proceed as follows: In the ensuing Section 2 the class of systems under consideration is defined and we prove some preliminary results. In Section 3 the optimal control problem that characterizes the domain of asymptotic null controllability is introduced and it is shown that under suitable conditions the corresponding value function is continuous, positive definite and proper on the domain of asymptotic null-controllability. In Section 4 we show that the value function of the optimal control problem is the unique viscosity solution of the generalized Zubov equation. 
In Section 5 we discuss an approximation of the problem with unbounded control set with a sequence of problems with bounded control set. In the last section we discuss the necessity of our assumptions at the hand of a few examples. It is also shown that for the classical linear quadratic control problem the general equations of this paper reduce to the standard algebraic Riccati equation.

\section{The DOMAin of Null CONTROLlability}

We consider nonlinear control systems of the type

$$
\dot{x}(t)=f(x(t), u(t))
$$

where $f: \mathbb{R}^{n} \times U \rightarrow \mathbb{R}$ is continuous, $U \subset \mathbb{R}^{m}$ is a closed set and the space of admissible control functions is given by

$$
u \in \mathcal{U}:=L^{\infty}([0, \infty), U)
$$

Solutions corresponding to an initial value $x$ and a control $u \in \mathcal{U}$ at time $t$ are denoted by $\varphi(t, x, u)$, which are defined on a maximal positive interval of definition $\left[0, T_{\max }(x, u)\right)$, where we do not exclude the case that $\left.T_{\max }(x, u)\right)<\infty$. i.e. that solutions explode. In the following the open ball of radius $r$ around a point $z \in \mathbb{R}^{p}$ is denoted by $B(z, r)$.

Uniqueness of solutions is a consequence of our further standard assumption on $f$ These are formulated using comparison functions, a fashionable approach these days. $^{1}$

There exists $\gamma \in \mathcal{K}_{\infty}$ such that for any $R>0$ there is $C_{R}>0$ with

$$
\|f(x, u)-f(y, u)\| \leq C_{R}(1+\gamma(\|u\|))\|x-y\|,
$$

for all $x, y$ with $\|x\|,\|y\| \leq R$,

$$
f(0,0)=0
$$

There exists an open ball $B(0, r)$, a constant $\bar{u}>0$, and $\beta \in \mathcal{K} \mathcal{L}$ such that for any $x \in B(0, r)$ there exists $u_{x} \in \mathcal{U}$ with $\left\|u_{x}\right\|_{\infty} \leq \bar{u}$, $T_{\max }\left(x, u_{x}\right)=\infty$ and

$$
\left\|\varphi\left(t, x, u_{x}\right)\right\| \leq \beta(\|x\|, t), \quad \forall t \geq 0 .
$$

Remark 2.1. The Lipschitz assumption (H0) is weaker than the following assumption:

For any $R>0$ there exists $C_{R}>0$ with

$$
\|f(x, u)-f(y, u)\| \leq C_{R}(1+\|u\|)\|x-y\|,
$$

for all $x, y$ with $\|x\|,\|y\| \leq R$,

Assumption (2.2) is used in many papers on viscosity solutions, in particular in $[33,34]$, whose results we will use later. In order to be able to use these results under the weaker assumption (H0) we define the map $R: \mathbb{R}^{m} \rightarrow \mathbb{R}^{m}$ by $R(u)=$ $\gamma^{-1}(\|u\|) u /\|u\|$ and consider the vector field

$$
\hat{f}(x, u)=f(x, R(u))
$$

\footnotetext{
${ }^{1}$ As usual we call a function $\alpha$ of class $\mathcal{K}_{\infty}$ if it is a homeomorphism of $[0, \infty)$, a continuous function $\beta$ in two real nonnegative arguments is called of class $\mathcal{K} \mathcal{L}$ if it is of class $\mathcal{K}_{\infty}$ in the first and decreasing to zero in the second argument.
} 
with $u \in \widetilde{U}:=R^{-1}(U)$. This input transformed system satisfies

$$
\|\hat{f}(x, u)-\hat{f}(y, u)\| \leq C_{R}(1+\gamma(\|R(u)\|))\|x-y\|=C_{R}(1+\|u\|)\|x-y\|,
$$

i.e., (2.2). Hence by applying the results from $[33,34]$ to $\hat{f}$ these immediately carry over to $f$ under the weaker assumption $(\mathrm{H} 0)$.

Property (H2) is a local asymptotic controllability property, which ensures that at least from a neighborhood of 0 the system may be steered to 0 .

For certain systems it makes sense to strengthen this local asymptotic controllability property $(\mathrm{H} 2)$ by requiring that $u_{x}$ is not only bounded but also converges to 0 as $t \rightarrow \infty$. In this case we can strengthen (H2) to the so-called small control property

There exists an open ball $B(0, r)$ and $\beta \in \mathcal{K} \mathcal{L}$ such that for any $x \in B(0, r)$ there exists $u_{x} \in \mathcal{U}$ with $T_{\max }\left(x, u_{x}\right)=\infty$ and

$$
\left\|\varphi\left(t, x, u_{x}\right)\right\|+\left\|u_{x}(t)\right\| \leq \beta(\|x\|, t), \quad \text { a.e. } t \geq 0 .
$$

Note that (H2') implies (H2) with $\bar{u}=\beta(r, 0)$. It is known [30] that for any $\beta \in \mathcal{K} \mathcal{L}$ there exist two functions $\alpha_{1}, \alpha_{2} \in \mathcal{K}^{\infty}$ such that $\beta(r, t) \leq \alpha_{2}\left(\alpha_{1}(r) e^{-t}\right)$. For ease of presentation we will work with these two functions from now on. Furthermore, we will from now on tacitly assume that $T_{\max }(x, u)=\infty$, if we write $\varphi(t, x, u) \rightarrow 0$ as $t \rightarrow \infty$.

We define the domain of null controllability by

$$
\mathcal{D}_{0}:=\left\{x \in \mathbb{R}^{n} \mid \text { there exists } u \in \mathcal{U} \text { with }\|\varphi(t, x, u)\| \rightarrow 0 \text { for } t \rightarrow \infty\right\},
$$

and the first hitting time with respect to $B(0, r)$ by

$$
t(x, u):=\inf \{t \geq 0 \mid \varphi(t, x, u) \in B(0, r)\},
$$

with the convention $\inf \emptyset=\infty$. The following lemma shows how $\mathcal{D}_{0}$ and $t(x, u)$ are related.

Lemma 2.2. The set $\mathcal{D}_{0}$ is given by

$$
\mathcal{D}_{0}=\left\{x \in \mathbb{R}^{n} \mid \inf _{u \in \mathcal{U}} t(x, u)<\infty\right\} .
$$

Proof. If we find $u \in \mathcal{U}$ with $t(x, u)<\infty$ then for some $t(x, u)<t_{1}$ we have $\varphi\left(t_{1}, x, u\right) \in B(0, r)$ and we can concatenate $\left.u\right|_{\left[0, t_{1}\right]}$ with the control $u_{\varphi\left(t_{1}, x, u\right)}$ from $(\mathrm{H} 1)$, which implies $\varphi(t, x, u) \rightarrow 0$. Hence we obtain

$$
\mathcal{D}_{0} \subseteq\left\{x \in \mathbb{R}^{n} \mid \inf _{u \in \mathcal{U}} t(x, u)<\infty\right\} .
$$

Conversely, if $x \in \mathcal{D}_{0}$ then we have $\varphi(t, x, u) \rightarrow 0$ for some suitable $u \in \mathcal{U}$, which implies $\varphi\left(t_{1}, x, u\right) \in B(0, r)$ for some $t_{1}>0$ and consequently $t(x, u) \leq t_{1}<\infty$ which implies the converse direction.

For the formulation of the next result recall that a set $M$ is called viable (or controlled or weakly invariant) if for every $x \in M$ there is a $u \in \mathcal{U}$ such that $\varphi(t, x, u) \in M$ for all $t \geq 0$. In the following the convex hull of a set $M$ is denoted by conv $M$.

Proposition 2.3. Assume (HO), (H1) and (H2) or (H2'). Then the following properties hold. 
(i) $\operatorname{cl} B(0, r) \subset \mathcal{D}_{0}$,

(ii) the set $\mathcal{D}_{0}$ is open, connected and viable.

Proof. (i): It is clear that $B(0, r) \subset \mathcal{D}_{0}$ so assume that for some $x \in \partial B(0, r)$ we have $x \notin \mathcal{D}_{0}$. Let $\left\{x_{n}\right\} \subset B(0, r)$ be a sequence with $\lim _{n \rightarrow \infty} x_{n}=x$. By assumption to each $x_{n}$ there exists a control $u_{n} \in \mathcal{U} \cap L^{\infty}(\mathbb{R}, B(0, \bar{u}))$ such that $\left\|\varphi\left(t, x_{n}, u_{n}\right)\right\| \leq \alpha_{2}\left(\alpha_{1}(r) e^{-t}\right)$. This shows that on each compact interval the solutions are bounded uniformly in $n$. Furthermore, they are equicontinuous by the boundedness of $f$ on $\mathrm{cl} B(0, r) \times(B(0, \bar{u}) \cap U)$. Thus by the Arzela-Ascoli theorem and a diagonal sequence argument we may choose a subsequence satisfying $x\left(\cdot, x_{n}, u_{n}\right) \rightarrow y(\cdot)$ uniformly on compact intervals. By [2, Theorem 2.2.1] it follows that $y$ is a solution of

$$
\dot{y} \in \operatorname{conv} f(y, U \cap B(0, \bar{u})) .
$$

By construction $\|y(t)\| \leq \alpha_{2}\left(\alpha_{1}(r) e^{-t}\right)$, so that $y(t) \in B(0, r / 2)$ for some $t$ large enough. Now by $\left[2\right.$, Theorem 2.4.2] there are controls $v_{n} \in \mathcal{U} \cap L^{\infty}(\mathbb{R}, B(0, \bar{u}))$ such that $x\left(\cdot, x, v_{n}\right)$ converges uniformly to $y$ on $[0, t]$. It follows that $x\left(t, x, v_{n}\right) \in B(0, r)$ for some $n$ large enough which shows that $x$ can be asymptotically steered to 0 .

(ii): Let $x_{0} \in \mathcal{D}_{0}$ and $u \in \mathcal{U}$ with $\varphi\left(t, x_{0}, u\right) \rightarrow 0$ for $t \rightarrow \infty$. Then there exists $T>0$ such that $\varphi\left(T, x_{0}, u\right) \in B(0, r)$. By continuous dependence on the initial value we obtain

$$
\varphi(T, x, u) \in B(0, r)
$$

for all $x$ in a neighborhood of $x_{0}$. Thus $t(\cdot, u)$ is finite on that neighborhood which shows that it is contained in $\mathcal{D}_{0}$. As $x_{0}$ was arbitrary this shows the assertion.

Since for any $x \in \mathcal{D}_{0}$ there exists a trajectory from $x$ to $B(0, r)$ we obtain that $\mathcal{D}_{0}$ is connected.

In order to see viability, consider a point $x \in \mathcal{D}_{0}$ and the trajectory $\varphi(t, x, u) \rightarrow 0$. Clearly, each point $x(t)=\varphi(t, x, u), t \geq 0$ can be controlled to the origin by the control $u(t+\cdot)$, thus $x(t) \in \mathcal{D}_{0}$ and hence $\varphi(t, x, u) \in \mathcal{D}_{0}$ for all $t \geq 0$, i.e., $\mathcal{D}_{0}$ is viable.

Remark 2.4. Note that the domain of nullcontrollability $\mathcal{D}_{0}$ is in general not diffeomorphic to $\mathbb{R}^{n}$. This is in contrast to the theory of domains of attraction of (perturbed) ordinary differential equations. In the case of asymptotically stable fixed points the domain of attraction is diffeomorphic to $\mathbb{R}^{n}$ even for perturbed systems, see e.g. $[8,36]$.

\section{Characterization of $\mathcal{D}_{0}$ using Optimal Control}

In this section we describe how to characterize the domain of asymptotic nullcontrollability via an optimal control problem and show continuity of the corresponding value function. In order to set up the problem we need a running cost $g: \mathbb{R}^{n} \times U \rightarrow \mathbb{R}$. The assumptions on $g$ are as follows:

The function $g: \mathbb{R}^{n} \times U \rightarrow \mathbb{R}$ is continuous and satisfies (H0) with the same $\gamma \in \mathcal{K}_{\infty}$ as $f$. Furthermore, for all $c>0$ we have

$$
\inf \{g(x, u) \mid\|x\| \geq c, u \in U\}=: g_{c}>0 \text {. }
$$

We need to ensure convergence of the integral cost that is introduced shortly for the "right" stabilizing solutions. To this end using the simplification $\beta(r, t) \leq$ $\alpha_{2}\left(\alpha_{1}(r) e^{-t}\right)$ for $\beta$ from (H2) and some arbitrary $\eta>0$ we assume that there is a constant $C>0$ such that 


$$
\begin{gathered}
g(x, u) \leq C\left(\alpha_{2}^{-1}(\|x\|)\right)^{\eta} \text { for all }(x, u) \in B(0, r) \times B(0, \bar{u}) . \\
g(x, u) \geq\|f(x, u)\|+\gamma(\|u\|) \text { whenever }\|x\| \geq 2 r \text { or }\|u\| \geq 2 \bar{u} .
\end{gathered}
$$

Remark 3.1. If the small control asymptotic controllability property (H2') holds then we can weaken assumption (H4) to

$$
g(x, u) \leq C\left(\alpha_{2}^{-1}(\|x\|+\|u\|)\right)^{\eta} \text { for all }(x, u) \in B(0, r) \times B(0, \bar{u}) .
$$

In what follows we will always assume that either (H2) and (H4) or (H2') and (H4') hold.

We now define the functional

$$
J(x, u):=\left\{\begin{array}{cc}
\int_{0}^{\infty} g(\varphi(t, x, u), u(t)) d t, & \text { if } T_{\max }(x, u)=\infty \\
\infty & \text { else }
\end{array},\right.
$$

the (extended real valued) optimal value function

$$
V(x):=\inf _{u \in \mathcal{U}} J(x, u), \quad x \in \mathbb{R}^{n},
$$

and the function

$$
v(x):=1-e^{-V(x)}, \quad x \in \mathbb{R}^{n} .
$$

Note that both $V$ and $v$ satisfy appropriate dynamic programming principles, i.e., for each $T>0$ we have

$$
V(x)=\inf _{u \in \mathcal{U}}\left\{\int_{0}^{T} g(\varphi(t, x, u), u(t)) d t+V(\varphi(T, x, u))\right\},
$$

and

$$
v(x)=\inf _{u \in \mathcal{U}}\{1+G(x, T, u)(v(\varphi(T, x, u))-1)\}
$$

where

$$
G(x, T, u):=\exp \left(-\int_{0}^{T} g(\varphi(t, x, u), u(t)) d t\right) .
$$

We now investigate the properties of $V$ and $v$. For this purpose we need the following observation on the solutions of (2.1). Using the function $\gamma$ from (H0) we define for $u \in \mathcal{U}$

$$
\|u\|_{\gamma, T}:=\int_{0}^{T} \gamma(\|u(t)\|) d t .
$$

Lemma 3.2. Let $T>0$. If $x \in \mathbb{R}^{n}$ and $u \in \mathcal{U}$ are such that $\|\varphi(t, x, u)\| \geq 2 r, t \in$ $[0, T]$ or $\|u(t)\| \geq 2 \bar{u}$ a.e. $t \in[0, T]$, then

$$
\int_{0}^{T} g(\varphi(t, x, u), u(t)) d t \geq\|\varphi(T, x, u)-x\|+\|u\|_{\gamma, T}
$$

Proof. Using (H5) we have that

$$
\int_{0}^{T} g(\varphi(t, x, u), u(t)) d t \geq \int_{0}^{T}\|f(\varphi(t, x, u), u(t))\| d t+\int_{0}^{T} \gamma(\|u(t)\|) d t,
$$

and the claim follows.

Proposition 3.3. Assume $(\mathrm{HO})-\left(\mathrm{H}_{4}\right)$ or $\left.(\mathrm{HO})-\mathrm{H}_{\left(\mathrm{2}^{\prime}\right)}\right)-\left(\mathrm{H}_{4}{ }^{\prime}\right)$. Then 
(i) the inequalities $V(x)<\infty$ and $v(x)<1$ hold if and only if $x \in \mathcal{D}_{0}$,

(ii) if in addition (H5) holds, then $V(x)=0 \Leftrightarrow x=0$ and $v(x)=0 \Leftrightarrow x=0$.

Proof. From the definition of $v$ it immediately follows that that the claims for $V$ and $v$ are equivalent. We show the statements for $V$.

(i) Pick a point $x \in \mathcal{D}_{0}$. Then there exists $u \in \mathcal{U}$ and $t_{1}>0$ such that $\left\|\varphi\left(t_{1}, x, u\right)\right\| \leq \alpha_{1}^{-1} \circ \alpha_{2}^{-1}(r)$. (Note that $\alpha_{1}^{-1} \circ \alpha_{2}^{-1}(r) \leq r$ follows from the definition of $\alpha_{1}, \alpha_{2}$.) By asumption (H1) we can assume (by changing $u$ on $\left[t_{1}, \infty\right.$ ) if necessary) that $\left\|\varphi\left(t_{1}+t, x, u\right)\right\| \leq \alpha_{2}\left(\alpha_{1}\left(\left\|\varphi\left(t_{1}, x, u\right)\right\|\right) e^{-t}\right) \leq r$ for all $t \geq 0$. Since $u \in \mathcal{U}=\mathrm{L}^{\infty}([0, \infty), U)$ is essentially bounded we can find $\tilde{u}>0$ such that $\|u(t)\| \leq \tilde{u}$ for almost all $t \geq 0$. Furthermore, by continuity of $\varphi(t, x, u)$ in $t$ we find $R>0$ such that $\|\varphi(t, x, u)\| \leq R$ for all $t \in\left[0, t_{1}\right]$. Hence using (H4) we can estimate

$$
\begin{aligned}
V(x) & \leq \int_{0}^{t_{1}} g(\varphi(t, x, u), u(t)) d t+\int_{t_{1}}^{\infty} g(\varphi(t, x, u), u(t)) d t \\
& \left.\leq t_{1} \sup _{x \in B(0, R), u \in B(0, \tilde{u})} g(x, u)+\int_{t_{1}}^{\infty} C\left(\alpha_{2}^{-1}(\| \varphi(t, x, u), u(t)) \|\right)\right)^{\eta} d t \\
& \leq t_{1} \sup _{x \in B(0, R), u \in B(0, \tilde{u})} g(x, u)+\frac{C}{\eta} \alpha_{1}\left(\left\|\varphi\left(t_{1}, x, u\right)\right\|\right)^{\eta}<\infty .
\end{aligned}
$$

If (H2') and (H4') hold, then the proof is completely analogous.

Conversely, let $x \notin \mathcal{D}_{0}$. Then we obtain $t(x, u)=\infty$ for all $u \in \mathcal{U}$ which implies

$$
J(x, u)=\int_{0}^{\infty} g(\varphi(t, x, u), u(t)) d t \geq \int_{0}^{\infty} g_{r} d t=\infty
$$

for each $u \in \mathcal{U}$ and thus also $V(x)=\inf _{u \in \mathcal{U}} J(x, u)=\infty$.

(ii) It is clear that $V(0)=0$, so let $x \neq 0$. Assume to the contrary that there is a sequence $\left\{u_{k}\right\} \subset \mathcal{U}$ such that $J\left(x, u_{k}\right) \rightarrow 0$. Let $c:=\|x\| / 2$ and denote

$$
t_{k}:=\inf \left\{t \geq 0 \mid\left\|\varphi\left(t, x, u_{k}\right)\right\| \leq c\right\} .
$$

By (H3) we have for all $k$ that $J\left(x, u_{k}\right) \geq \int_{0}^{t_{k}} g\left(\varphi\left(s, x, u_{k}\right), u_{k}(s)\right) d s \geq t_{k} g_{c}$ which implies that $t_{k} \rightarrow 0$. Now $\|f\|$ is bounded on $B(0,2 r) \times B(0,2 \bar{u})$ by the constant $C:=C_{2 r}(1+\gamma(2 \bar{u})) 2 r$. Denote

$$
E(k):=\left\{t \in\left[0, t_{k}\right] \mid\left(\varphi\left(t, x, u_{k}\right), u(t)\right) \in B(0,2 r) \times B(0,2 \bar{u})\right\},
$$

which is well defined up to a set of measure zero. Then

$$
\int_{E(k)}\left\|f\left(\varphi\left(t, x, u_{k}\right), u_{k}(t)\right)\right\| d t \leq t_{k} C .
$$

On the other hand we have for all $k$ that

$$
\int_{0}^{t_{k}}\left\|f\left(\varphi\left(t, x, u_{k}\right), u_{k}(t)\right)\right\| d t \geq\left\|x-\varphi\left(t_{k}, x, u_{k}\right)\right\| \geq c .
$$

Using (H5) this implies that

$$
\begin{gathered}
J\left(x, u_{k}\right) \geq \int_{\left[0, t_{k}\right] \backslash E(k)} g\left(\varphi\left(s, x, u_{k}\right), u_{k}(s)\right) d s \\
\geq \int_{\left[0, t_{k}\right] \backslash E(k)}\left\|f\left(\varphi\left(s, x, u_{k}\right), u_{k}(s)\right)\right\| d s \geq c-t_{k} C .
\end{gathered}
$$

As $t_{k} \rightarrow 0$ this contradicts $J\left(x, u_{k}\right) \rightarrow 0$. 
Next we turn to the investigation of the regularity properties of the functions $V$ and $v$. We start by proving continuity properties for the trajectories of (2.1).

Lemma 3.4. Assume (HO) and let $T>0$ and $R>0$ be arbitrary constants. Then for all $x, y \in \mathbb{R}^{n}$ and all $u \in \mathcal{U}$ satisfying

$$
\|\varphi(t, x, u)\| \leq R, \quad\|\varphi(t, y, u)\| \leq R, \quad \forall t \in[0, T]
$$

we have

$$
\|\varphi(t, x, u)-\varphi(t, y, u)\| \leq e^{C_{R}\left(\|u\|_{\gamma, t}+t\right)}\|x-y\|,
$$

for all $t \in[0, T]$.

Proof. The assumption (H0) yields for almost all $t \in[0, T]$

$$
\begin{aligned}
& \|f(\varphi(t, x, u), u(t))-f(\varphi(t, y, u), u(t))\| \leq \\
& C_{R}(1+\gamma(\|u(t)\|))\|\varphi(t, x, u)-\varphi(t, y, u)\| .
\end{aligned}
$$

Using (3.8) Gronwall's Lemma we then obtain

$$
\|\varphi(t, x, u)-\varphi(t, y, u)\| \leq e^{C_{R}\left(\int_{0}^{t}(1+\gamma(\|u\|)) d t\right)}\|x-y\|,
$$

and the assertion follows.

Using this lemma we can prove the following continuity statement.

Proposition 3.5. Assume (HO)-(H5), where (H2) and (H4) can be replaced by (H2') and (H4'). Then $V$ and $v$ are continuous on $\mathcal{D}_{0}$.

Proof. We show the continuity of $V$, then the statement for $v$ follows immediately from its definition. The proof is performed in several steps. Throughout the proof the constants $C_{R}, C$ etc. are those defined in (H0) and (H4), resp. (H4').

First note that from (3.6) we have

$$
V(x) \leq \frac{C}{\eta} \alpha_{1}(\|x\|)^{\eta}, \quad \text { for } x \in B\left(0, \alpha_{1}^{-1} \circ \alpha_{2}^{-1}(r)\right) .
$$

(i) (Local boundedness of $V$ on $\mathcal{D}_{0}$ )

Pick an arbitrary $x_{0} \in \mathcal{D}_{0}$ and fix $\varepsilon>0$. Then there exists a $u_{0} \in \mathcal{U}$ such that $J\left(x_{0}, u_{0}\right) \leq V\left(x_{0}\right)+\varepsilon$. Since $J\left(x_{0}, u_{0}\right)$ is finite it follows from (H3) there exists a time $T_{0}>0$ such that $\left\|\varphi\left(T_{0}, x_{0}, u_{0}\right)\right\| \leq \alpha_{1}^{-1} \circ \alpha_{2}^{-1}(r) / 2$. By continuity of $\varphi$ in $x$ we can pick a ball $B\left(x_{0}, \delta\right)$ such that

$$
\left\|\varphi\left(T_{0}, x, u_{0}\right)\right\| \leq \alpha_{1}^{-1} \circ \alpha_{2}^{-1}(r), \quad \text { for all } x \in \operatorname{cl} B\left(x_{0}, \delta\right) .
$$

We define the set

$$
K=\left\{\varphi\left(t, x, u_{0}\right) \mid x \in \operatorname{cl} B\left(x_{0}, \delta\right), t \in\left[0, T_{0}\right]\right\}
$$

which is compact since $\varphi$ is continuous in $t$ and $x$ (recall that $u_{0}$ is essentially bounded). Using (3.10) we obtain from Bellman's optimality principle for all $x \in$ $B\left(x_{0}, \delta\right)$ the inequality

$$
\begin{aligned}
V(x) \leq & \int_{0}^{T_{0}} g(\varphi(t, x, u), u(t)) d t+V\left(\varphi\left(T_{0}, x, u\right)\right) \\
& \leq \max _{x \in K, u \in B\left(0,\left\|u_{0}\right\|_{\infty}\right)} g(x, u) T+\frac{C}{\eta} \alpha_{1}(r)^{\eta},
\end{aligned}
$$

where we have used (3.9). This shows that $\sup _{x \in B\left(x_{0}, \delta\right)} V(x)=: B_{V}$ is finite.

(ii) (Bounds on $\varepsilon$-optimal controls and trajectories) 
For any $x \in B\left(x_{0}, \delta\right)$ and any $\varepsilon \in(0,1]$ we pick an $\varepsilon$-optimal control function $u_{x, \varepsilon} \in \mathcal{U}$, i.e.,

$$
J\left(x, u_{x, \varepsilon}\right) \leq V(x)+\varepsilon .
$$

We claim that for any $\varepsilon, T>0$ the set

$$
K_{\varepsilon}:=\left\{\varphi\left(t, x, u_{x, \varepsilon}\right) \mid t \geq 0, x \in B\left(x_{0}, \delta\right)\right\}
$$

and the sets

$$
\left\{\left\|u_{x, \varepsilon}\right\|_{\gamma, T} \mid x \in B\left(x_{0}, \delta\right)\right\}
$$

are bounded. If the first set were unbounded then there would be an $x \in B\left(x_{0}, \delta\right)$ and $t_{1}>0$ such that $\left\|\varphi\left(t_{1}, x, u_{x, \varepsilon}\right)\right\| \geq V(x)+2 \varepsilon+2 r$. If $t_{2}>t_{1}$ is the first time at which $\left\|\varphi\left(t_{1}, x, u_{x, \varepsilon}\right)\right\|=2 r$ again, then we obtain using Lemma 3.2 that

$J\left(x, u_{x, \varepsilon}\right) \geq \int_{t_{1}}^{t_{2}} g\left(\varphi\left(t, x, u_{x, \varepsilon}\right), u_{x, \varepsilon}(t)\right) d t \geq\left\|\varphi\left(t_{1}, x, u_{x, \varepsilon}\right)-\varphi\left(t_{2}, x, u_{x, \varepsilon}\right)\right\| \geq V(x)+2 \varepsilon$,

a contradiction.

On the other hand, if $\left\{\left\|u_{x, \varepsilon}\right\|_{\gamma, T} \mid x \in B\left(x_{0}, \delta\right)\right\}$ is unbounded for a given $T>0$, then there have to be $x, u_{x, \varepsilon}$ such that $\left\|u_{x, \varepsilon}\right\|_{\gamma, T} \geq V(x)+2 \varepsilon+T \gamma(2 \bar{u})$. This implies that if we integrate over the (measurable) set

$$
E:=\left\{t \in[0, T] \mid\left\|u_{x, \varepsilon}(t)\right\| \geq 2 \bar{u}\right\},
$$

then we obtain

$$
\int_{E} \gamma\left(\left\|u_{x, \varepsilon}(t)\right\|\right) d t \geq V(x)+2 \varepsilon,
$$

as the contribution of the integral over $[0, T] \backslash E$ to $\left\|u_{x, \varepsilon}\right\|_{\gamma, T}$ can be at most $T \gamma(2 \bar{u})$. Using an estimate over the set $E$ and again Lemma 3.2 we obtain again a contradiction to $J\left(x, u_{x, \varepsilon}\right) \leq V(x)+\varepsilon$.

(iii) (Continuity of trajectories)

We denote by $R_{\varepsilon}$ an upper bound on the set $K_{\varepsilon}$. By Lemma 3.4 we can conlude that for $x, y \in B\left(x_{0}, \delta\right)$ and all $t \geq 0$ such that

$$
\|x-y\| \leq R_{\varepsilon} \exp \left(-C_{2 R_{\varepsilon}}\left(\left\|u_{x, \epsilon}\right\|_{\gamma, t}+t\right)\right)
$$

we have

$$
\left\|\varphi\left(t, x, u_{x, \epsilon}\right)-\varphi\left(t, y, u_{x, \epsilon}\right)\right\| \leq \exp \left(C_{2 R_{\varepsilon}}\left(\left\|u_{x, \varepsilon}\right\|_{\gamma, t}+t\right)\right)\|x-y\| .
$$

(iv) (Continuity of $V$ )

We show the continuity of $V$ on $B\left(x_{0}, \delta\right)$. Since $x_{0} \in \mathcal{D}_{0}$ was arbitrary this proves the proposition. So pick $\varepsilon>0$ and assume without loss of generality that $\epsilon<\alpha_{2}^{-1}(r) C$.

From the lower bound $g_{c}$ on $g$ in (H3) and the boundedness of $J\left(x, u_{x, \varepsilon}\right)$ on $B\left(x_{0}, \delta\right)$ it follows that for any $\rho>0$ there is a time $T_{\rho}$ such that for $x \in B\left(x_{0}, \delta\right)$ we have $\varphi\left(t, x, u_{x, \varepsilon}\right) \in B(0, \rho)$ for some $t \leq T_{\rho}$. Using (3.9) we may thus assume that the controls $u_{x, \varepsilon}$ are chosen in such a way that there exists $T_{\epsilon}>0$ (depending on $\left.B_{V}\right)$ such that for all $t \geq T_{\epsilon}, x \in B(0, \delta)$ we have

$$
\varphi\left(t, x, u_{x, \varepsilon}\right) \in B\left(0, \alpha_{1}^{-1}(\varepsilon / C) / 2\right) \subset B\left(0, \alpha_{1}^{-1} \circ \alpha_{2}^{-1}(r) / 2\right) .
$$

Denote

$$
m:=\exp \left(-C_{2 R_{\varepsilon}}\left(\max _{z \in B\left(x_{0}, \delta\right)}\left\|u_{z, \epsilon}\right\|_{\gamma, T_{\varepsilon}}+T_{\varepsilon}\right)\right)
$$


and note that the right hand side is finite by (ii). Choose two points $x, y \in B\left(x_{0}, \delta\right)$ such that

$$
\|x-y\| \leq R_{\varepsilon} m \text {. }
$$

Without loss of generality assume $V(y) \geq V(x)$. Abbreviating $u:=u_{x, \varepsilon}, T:=T_{\varepsilon}$ we obtain

$$
\begin{aligned}
& |V(y)-V(x)|=V(y)-V(x) \\
& \quad \leq V(y)-\int_{0}^{\infty} g(\varphi(t, x, u), u(t)) d t+\varepsilon \\
& \quad \leq \int_{0}^{T}|g(\varphi(t, y, u), u(t))-g(\varphi(t, x, u), u(t))| d t+V(\varphi(T, y, u))+\varepsilon
\end{aligned}
$$

using the Lipschitz condition in (H3) and (3.11) we continue

$$
\leq \int_{0}^{T} C_{2 R_{\epsilon}}(1+\gamma(\|u(t)\|)) m\|x-y\| d t+V(\varphi(T, y, u))+\varepsilon
$$

and we obtain

$$
\leq C_{2 R_{\varepsilon}}\left(T+\|u\|_{\gamma, T}\right) m\|x-y\|+2 \varepsilon,
$$

provided $\|y-x\| \leq \alpha_{1}^{-1}\left(\eta \varepsilon^{1 / \eta} / C\right) /(2 m)$, because in this case we obtain from (3.11) that $\varphi(T, y, u) \in B\left(0, \alpha_{1}^{-1}\left(\eta \varepsilon^{1 / \eta} / C\right)\right)$ and thus from $(3.9)$

$$
V(\varphi(T, y, u)) \leq \frac{C}{\eta} \alpha_{1}(\|\varphi(T, y, u)\|)^{\eta} \leq \varepsilon .
$$

Thus for any $\varepsilon \in(0,1]$ and any $x \in B\left(x_{0}, \delta\right)$ we can find $\delta_{\varepsilon}>0$ such that $\mid V(y)-$ $V(x) \mid \leq 3 \varepsilon$, for all $x, y \in B\left(x_{0}, \delta\right)$ with $\|x-y\| \leq \delta_{\varepsilon}$. This implies continuity of $V$ in $B\left(x_{0}, \delta\right)$ and, since $x_{0} \in \mathcal{D}_{0}$ was arbitrary, continuity on the whole set $\mathcal{D}_{0}$.

The next proposition makes a statement of the behavior of $V(x)$ near the boundary of $\mathcal{D}_{0}$ or at $\infty$.

Proposition 3.6. Assume (HO)-(H5) or their respective variants from Remark 3.1. Then for any sequence $x_{k}$ which satisfies $\operatorname{dist}\left(x_{k}, \partial \mathcal{D}_{0}\right) \rightarrow 0$ or $\left\|x_{k}\right\| \rightarrow \infty$ we have $V\left(x_{k}\right) \rightarrow \infty$ and $v\left(x_{k}\right) \rightarrow 1$. In particular, $v$ is continuous on $\mathbb{R}^{n}$.

Proof. If $\left\|x_{k}\right\| \rightarrow \infty$, then we have for every $n$ either that $x_{k} \notin \mathcal{D}_{0}$, in which case $V\left(x_{k}\right)=\infty$ or $x_{k} \in \mathcal{D}_{0}$. In the latter case we have by Lemma 3.2 that $V\left(x_{k}\right) \geq\left\|x_{k}\right\|-2 r$, for all $k$ large enough. This shows the assertion for $V$ and the conclusion for $v$ is immediate from the definition.

To prove the assertion for $\operatorname{dist}\left(x_{k}, \partial \mathcal{D}_{0}\right) \rightarrow 0$, we may now assume that there exists a sequence $x_{k} \rightarrow x_{0} \in \partial \mathcal{D}_{0}$ and some $C>0$ such that $V\left(x_{k}\right) \leq C$ holds for all $k \in \mathbb{N}$. Pick $\varepsilon>0$ and for each $k$ choose a control function $u_{k} \in \mathcal{U}$ such that we have

$$
J\left(x_{k}, u_{k}\right) \leq V\left(x_{k}\right)+\varepsilon \leq C+\varepsilon .
$$

Following Step (ii) of the proof of Proposition 3.5 we obtain that $\left\{\varphi\left(t, x_{k}, u_{k}\right) \mid t \geq\right.$ $0, k \in \mathbb{N}\}$ is bounded and that $\left\|u_{k}\right\|_{\gamma, t}$ is uniformly bounded in $k$ for all $t \geq 0$. Then we may apply (3.11) as in Step (iv) of the proof of Proposition 3.5 to conclude that for every $t \geq 0$ and every $\delta>0$ there is a $k_{0}$ such that $\left\|\varphi\left(t, x_{k}, u_{k}\right)-\varphi\left(t, x_{0}, u_{k}\right)\right\|<\delta$ for all $k \geq k_{0}$. 
Because of the lower bound on $g$ in (H3) we may assume that there exists $T>0$ (independent of $n$ ) such that

$$
\varphi\left(t, x_{k}, u_{k}\right) \in B(0, r / 2) \text { for all } t \geq T, k \in \mathbb{N} .
$$

This implies $\varphi\left(T, x_{0}, u_{k}\right) \in B(0, r / 2)$ for all sufficiently large $k \in \mathbb{N}$ which in turn implies $x_{0} \in \mathcal{D}_{0}$. This contradicts $x_{0} \in \partial \mathcal{D}_{0}$ because $\mathcal{D}_{0}$ is open.

\section{Characterizations of $V$ And $v$ By Zubov's Method}

The aim of this section is to characterize the functions $V$ and $v$ introduced in (3.2) and (3.3) as (the unique) viscosity solutions of the equations

$$
\sup _{u \in U}\{-D V(x) f(x, u)-g(x, u)\}=0
$$

and

$$
\sup _{u \in U}\{-D v(x) f(x, u)-(1-v(x)) g(x, u)\}=0,
$$

respectively (for the definition of viscosity solution we refer to $[6,4]$ ).

Recalling that $V$ is locally bounded in $\mathcal{D}_{0}$ and $v$ is bounded in $\mathbb{R}^{n}$, our first result follows from a standard application of the dynamic programming principles (3.4) and (3.5), see [4].

Proposition 4.1. Assume (HO) - (H5) or their respective variants. Then the functions $V$ and $v$ defined in (3.2) and (3.3) are viscosity solutions of (4.1) in $\mathcal{D}_{0}$ and of of (4.2) in $\mathbb{R}^{n}$, respectively.

Remark 4.2. Note that it follows from these characterizations that $v$ is a control Lyapunov function on $\mathcal{D}_{0}$ in the usual sense, [32]. In fact, a small calculation shows that $v$ is a viscosity supersolution on $\mathcal{D}_{0}$ of

$$
\inf _{u \in U} D v(x) f(x, u) \leq-W(x) g_{\|x\|},
$$

where $0<W(x)<1-v(x)$ for $x \in \mathcal{D}_{0} \backslash\{0\}$ and $g_{\|x\|}$ denotes the constant from (H3) for $c=\|x\|$.

The main result in this section will be a uniqueness statement for the equations (4.1) and (4.2), showing that the above functions are the unique viscosity solutions of these equations.

In order to obtain such a result we make use of the so called optimality principles developed by Soravia [33, 34]. For the application of the results from these references we need that our system is defined by a bounded vector field $f$. To this end we introduce the following rescaled functions.

$$
\begin{gathered}
\tilde{f}(x, u)=\frac{f(x, u)}{1+\|f(x, u)\|}, \\
\tilde{g}(x, u)=\frac{g(x, u)}{1+\|f(x, u)\|} .
\end{gathered}
$$

Remark 4.3. The introduction of the vector field $\tilde{f}$ and the running cost $\tilde{g}$ amounts to nothing more than a rescaling of time, that does not change trajectories or values 
associated to a particular control. To see this let $x \in \mathbb{R}^{n}, u \in \mathcal{U}$ be given. Now introduce a new time variable $\tau$ through the differential equation

$$
\frac{d t(\tau)}{d \tau}=\frac{1}{1+\|f(\phi(t(\tau), x, u), u(t(\tau)))\|}, \text { a.e., }
$$

and a control $\tilde{u}(\tau):=u(t(\tau))$, a.e. Then the function $\psi(\tau):=\phi(t(\tau), x, u)$ satisfies the differential equation

$$
\frac{d \psi(\tau)}{d \tau}=\frac{f(\phi(t(\tau), x, u), u(t(\tau)))}{1+\|f(\phi(t(\tau), x, u), u(t(\tau)))\|}=\tilde{f}(\psi(\tau), \tilde{u}(\tau)) .
$$

So if we consider the system

$$
\dot{x}(t)=\tilde{f}(x(t), u(t)),
$$

it is easy to see that if $f$ satisfies (H0), (H1) and (H2) or (H2') then also $\tilde{f}$ satisfies these properties for suitably adjusted functions $\tilde{\gamma}$ and $\tilde{\beta}$. Also the domain of asymptotic nullcontrollability $\mathcal{D}_{0}$ is the same for the systems defined through $f$ and $\tilde{f}$.

Finally note, that $\tilde{g}$ clearly satisfies (H4) with a modified decay $\tilde{\alpha}_{2}$, as well as the first statement of (H3), however, the lower bound $g_{c}$ from (H3) need not exist because of the division by $1+\|f(x, u)\|$. Hence below we will impose this property as an additional assumption.

Consider now the optimal control problem for system (4.4) given by the running $\operatorname{cost} \tilde{g}$. If we assume that both the original as well as the rescaled functions satisfy (H0)-(H4) then using standard transformation of integral formulas it is also easy to see that if $T(x, u)=\infty$ then $\tilde{J}(x, \tilde{u})=J(x, u)$, where $\tilde{J}$ defines the value along a trajectory using the running cost $\tilde{g}$ in the spirit of (3.1). If the solution explodes, i.e. $T(x, u)<\infty$ then we have so far simply defined the value to be infinity. However, if we assume (H3) for $\tilde{g}$, then the associated integral of the transformed system also diverges. In all, this implies the value functions $v$ and $V$ coincide for the optimal control problems defined through (2.1) and (3.1), respectively (4.4) and using the running cost $\tilde{g}$ provided it satisfies (H3).

Now we introduce the necessary assumption for our uniqueness statement.

The rescaled function $\tilde{g}$ satisfies (H3) and, in addition, $\tilde{g}(x, u) \rightarrow \infty$ as $\|u\| \rightarrow \infty$ for each $x \in \mathbb{R}^{n}$.

To Zubov's equations (4.1) and (4.2) we associate the Hamiltonians

$$
H_{V}: \mathbb{R}^{n} \times\left(\mathbb{R}^{n}\right)^{*} \rightarrow \mathbb{R}, \quad H_{V}(x, p)=\sup _{u \in U}\{-f(x, u) p-g(x, u)\},
$$

and

$$
H_{v}: \mathbb{R}^{n} \times \mathbb{R} \times\left(\mathbb{R}^{n}\right)^{*} \rightarrow \mathbb{R}, \quad H_{v}(x, r, p)=\sup _{u \in U}\{-f(x, u) p-(1-r) g(x, u)\} .
$$

From (H5) we obtain that the supremum in these Hamiltonians is attained in a compact subset of $U$ for $r<1$ in the case of $H_{v}$. This implies that the Hamiltonians $H_{V}$ and $H_{v}$ are locally Lipschitz continuous with respect to their arguments, again for $r<1$ in the case of $H_{v}$.

In this section, we will prove the following main theorem of our paper.

Theorem 4.4. Assume that $f$ and $g$ satisfy the assumptions (HO)-(H6) (or their respective variants from Remark 3.1). Then 
(i) The function $v$ from (3.3) is the unique bounded viscosity solution of (4.2) with $v(0)=0$

(ii) There exists a unique couple $(\mathcal{O}, V)$ such that $\mathcal{O}$ is an open set containing the origin and $V$ is a locally bounded, continuous viscosity solution of (4.1) in $\mathcal{O}$ with $V(0)=0$ and $V(x) \rightarrow+\infty$ for $x \rightarrow \partial \mathcal{O}$. Here $V$ is the function from $(3.2)$.

(iii) The functions $v$ and $V$ characterize the domain of asymptotic controllability via

$$
\mathcal{D}_{0}=\left\{x \in \mathbb{R}^{n} \mid v(x)<1\right\}=\left\{x \in \mathbb{R}^{n} \mid V(x)<\infty\right\} .
$$

(iv) The functions $v$ and $V$ satisfy $v\left(x_{k}\right) \rightarrow 1$ and $V\left(x_{k}\right) \rightarrow \infty$ for all sequences with $x_{k} \rightarrow \partial \mathcal{D}_{0}$ or $\left\|x_{k}\right\| \rightarrow \infty$.

Note that the condition (H6) in this theorem is on $\tilde{g}$ rather than on $g$. One way in order to find such a $g$ would be to construct a suitable function $\bar{g}(x, u)$ meeting the assumptions (H0)-(H5) for $f$ and then define $g:=\bar{g}(1+\|f\|)$. In fact, this is very similar to Zubov's original approach, except that here we use the factor $1+\|f\|$ instead of $\sqrt{1+\|f\|^{2}}$.

In the proof of Theorem 4.4 we encounter two difficulties: the unbounded dependence of the functions on the control variable and the vanishing of the cost $g$ at the origin.

To solve the first problem we use the rescaled functions from above. Associated to these functions we introduce two rescaled equations which share with (4.1) and (4.2) the same set of sub- and supersolutions.

Lemma 4.5. Assume (HO) for $g$ and $\tilde{g}$ and consider the equations

$$
\sup _{u \in U}\{-D \tilde{V}(x) \tilde{f}(x, u)-\tilde{g}(x, u)\}=0
$$

and

$$
\sup _{u \in U}\{-D \tilde{v}(x) \tilde{f}(x, u)-(1-\tilde{v}(x)) \tilde{g}(x, u)\}=0 .
$$

Then

(i) Any viscosity subsolution of (4.1) is a viscosity subsolution for (4.5) and vice versa.

(ii) Any viscosity supersolution of (4.1) is a viscosity supersolution for (4.5), and, if in addition (H6) holds for $\tilde{g}$, then any viscosity supersolution of (4.5) is also a viscosity supersolution for (4.1).

The same assertions hold for (4.6) and (4.2).

Proof. We prove the lemma for (4.1) and (4.5), the assertions for (4.2) and (4.6) follow by the same arguments.

(i) If $V^{-}$is a viscosity subsolution of (4.1), then for any supergradient $p$ of $V^{-}$ in $x$ we have that

$$
\sup _{u \in U}\{-f(x, u) p-g(x, u)\} \leq 0
$$

This implies

$$
-f(x, u) p-g(x, u) \leq 0 \text { for all } u \in U
$$

and since $1+\|f(x, u)\|$ is positive, this implies

$$
-\tilde{f}(x, u) p-\tilde{g}(x, u)=(1+\|f(x, u)\|)^{-1}(-f(x, u) p-g(x, u)) \leq 0 \text { for all } u \in U
$$


which in turn implies

$$
\sup _{u \in U}\{-\tilde{f}(x, u) p-\tilde{g}(x, u)\} \leq 0,
$$

hence $V^{-}$is a viscosity supersolution of (4.5).

The converse direction follows by the same argument, since again we multiply by a positive factor, now $1+\|f(x, u)\|$.

(ii) Let $V^{+}$be a viscosity supersolution of (4.1). Then for any subgradient $p$ of $V^{+}$in $x$ we have

$$
\sup _{u \in U}\{-f(x, u) p-g(x, u)\} \geq 0 .
$$

Now we distinguish two cases:

(a) We can find $u^{*} \in U$ such that

$$
-f\left(x, u^{*}\right) p-g\left(x, u^{*}\right) \geq 0
$$

Since $1+\left\|f\left(x, u^{*}\right)\right\|$ is positive we obtain

$$
-\tilde{f}\left(x, u^{*}\right) p-\tilde{g}\left(x, u^{*}\right)=\left(1+\left\|f\left(x, u^{*}\right)\right\|\right)^{-1}\left(-\tilde{f}\left(x, u^{*}\right) p-\tilde{g}\left(x, u^{*}\right)\right) \geq 0 .
$$

This implies

$$
\sup _{u \in U}\{-\tilde{f}(x, u) p-\tilde{g}(x, u)\} \geq 0
$$

hence $V^{+}$is a viscosity supersolution of (4.5).

(b) For all $u \in U$ the inequality

$$
-f(x, u) p-g(x, u) \leq 0
$$

holds. In this case, since $1+\|f(x, u)\| \geq 1$, for all $u \in U$ we obtain

$$
\begin{aligned}
-\tilde{f}(x, u) p-\tilde{g}(x, u) & =\underbrace{(1+\|f(x, u)\|)^{-1}}_{\leq 1} \underbrace{(-f(x, u) p-g(x, u))}_{\leq 0} \\
& \geq-f(x, u) p-g(x, u) .
\end{aligned}
$$

This implies

$$
\sup _{u \in U}\{-\tilde{f}(x, u) p-\tilde{g}(x, u)\} \geq \sup _{u \in U}\{-f(x, u) p-g(x, u)\} \geq 0 .
$$

Thus also in this case $V^{+}$is a viscosity supersolution of (4.5).

Conversely, let $V^{+}$be a viscosity supersolution of (4.5). Then for any subgradient $p$ of $V^{+}$in $x$ we have

$$
\sup _{u \in U}\{-\tilde{f}(x, u) p-\tilde{g}(x, u)\} \geq 0 .
$$

Since $\tilde{f}$ is bounded and $\tilde{g}$ grows unbounded in $u$ due to (H6), the supremum over $u$ is contained in a compact set. Hence by continuity we can find a control value $u^{*} \in U$ for which the maximum is attained, i.e.

$$
-\tilde{f}\left(x, u^{*}\right) p-\tilde{g}\left(x, u^{*}\right) \geq 0 .
$$

Since $1+\left\|f\left(x, u^{*}\right)\right\|$ is positive we obtain

$$
-f\left(x, u^{*}\right) p-g\left(x, u^{*}\right)=\left(1+\left\|f\left(x, u^{*}\right)\right\|\right)\left(-\tilde{f}\left(x, u^{*}\right) p-\tilde{g}\left(x, u^{*}\right)\right) \geq 0 .
$$

This implies

$$
\sup _{u \in U}\{-f(x, u) p-g(x, u)\} \geq 0
$$

hence $V^{+}$is a viscosity supersolution of (4.1).

The following corollary is a simple consequence of this lemma. 
Corollary 4.6. Assume (HO) for $f$ and $\tilde{f}$ and (H6) for $\tilde{g}$. Then

(i) Any viscosity solution of (4.1) is a viscosity solution of (4.5) in $\mathcal{D}_{0}$ and vice versa.

(ii) Any viscosity solution of (4.2) is a viscosity solution of (4.6) and vice versa.

Even if the coefficients of the rescaled equations have a better dependence on the variable $u$, there is still the problem of the vanishing of $\tilde{g}$ at the origin. In order to prove a uniqueness result for (4.5) and (4.6), we use a control theoretic argument and some optimality principles introduced in $[33,34]$, as stated in the following lemma.

Lemma 4.7. Assume (HO) and (H3) for $\tilde{f}$ and $\tilde{g}$ and let $\tilde{\varphi}(t, x, u)$ be the solution of (4.4). Define

$$
\tilde{G}(x, t, u):=\exp \left(-\int_{0}^{t} \tilde{g}(\tilde{\varphi}(\tau, x, u), u(\tau)) d \tau\right) .
$$

Then the following properties hold.

(i) Any upper semicontinuous viscosity subsolution $w^{-}$of (4.6) satisfies

$$
w^{-}(x) \leq \inf _{u \in \mathcal{U}} \inf _{t \in[0, T]}\left\{1+\tilde{G}(x, t, u)\left(w^{-}(\tilde{\varphi}(t, x, u))-1\right)\right\} .
$$

for each $T>0$.

(ii) Consider a continuous viscosity supersolution $w^{+}$of (4.6) and let $\Omega \subset \mathbb{R}^{n}$ be an open and bounded set with $\sup _{x \in \Omega} w^{+}(x)<1$. Consider the first exit time from $\Omega$ given by

$$
T_{e x}(x, u, \Omega)=\min \left\{t \geq 0 \mid \varphi\left(t, x_{0}, u\right) \notin \Omega\right\} .
$$

Then $w^{+}$satisfies

$$
w^{+}(x) \geq \inf _{u \in \mathcal{U}} \sup _{t \in\left[0, T_{e x}(x, u, \Omega)\right]}\left\{1+\tilde{G}(x, t, u)\left(w^{+}(\tilde{\varphi}(t, x, u))-1\right)\right\} .
$$

Proof. Let $\Omega \subset \mathbb{R}^{n}$ be an open and bounded set and let $\widetilde{U}$ be a compact subset of $U$ with the corresponding space of measurable control functions denoted by $\widetilde{\mathcal{U}}$. If $w^{-}$is an upper semicontinuous viscosity subsolution of (4.6) in $\mathbb{R}^{n}$, then the restriction of $w^{-}$to $\Omega$ is also a subsolution of (4.6) on $\Omega$ with $\widetilde{U}$ instead of $U$. For the restricted control value set $\widetilde{U}$ equation 4.6 is continuous, furthermore $\tilde{f}, \tilde{g}$ are uniformly Lipschitz on $\Omega$. Hence we can apply [34, Theorem 3.2 (i)] which for each $u \in \widetilde{\mathcal{U}}$ yields

$$
w^{-}(x) \leq \inf _{t \in\left[0, T_{e x}(x, u, \Omega)\right]}\left\{1+\tilde{G}(x, t, u)\left(w^{-}(\tilde{\varphi}(t, x, u))-1\right)\right\},
$$

where $T_{e x}(x, u, \Omega)$ is the first exit time of $\tilde{\varphi}\left(t, x_{0}, u\right)$ from the set $\Omega$ defined in (ii).

Since $\tilde{f}$ is globally bounded, for any $x \in \mathbb{R}^{n}$ and any $T>0$ we may find an open and bounded set $\Omega_{x, T} \subset \mathbb{R}^{n}$ such that $T_{e x}\left(x, u, \Omega_{x, T}\right) \geq T$ for each $u \in \mathcal{U}$. Since each $u \in \mathcal{U}$ is essentially locally bounded, it lies in $\widetilde{\mathcal{U}}$ for an appropriate choice of $\widetilde{U}$, which shows (i).

The proof of (ii) follows from [34, Theorem 3.2 (ii)] observing that the equation (4.6) is continuous on $\Omega$ since $w^{-}(x)<1$, hence here we do not need to restrict the control value set $U$. 
Remark 4.8. Note that the asymmetry of the statements (i) and (ii) is due to the fact that we imposed different conditions in order to obtain continuity of (4.6), which is needed for the application of [34, Theorem 3.2]. In (i) we restrict the set of control values $U$ obtaining a result for arbitrary $\Omega$ (thus for arbitrary $T$ ) and for upper semicontinuous functions. In (ii) this restriction is not possible because the supersolution property will not persists passing from $U$ to $\widetilde{U}$. Thus here we ensure continuity of (4.6) by considering suitable subsets $\Omega$ of the state space.

Using these inequalities we can now prove the following uniqueness results.

Lemma 4.9. Assume (HO) - (H6) and consider the functions $V$ and $v$ defined by (3.2) and (3.3). Then

(i) $v$ is the unique bounded continuous viscosity solution of (4.6) with $v(0)=0$,

(ii) $\left(\mathcal{D}_{0}, V\right)$ is the unique couple of an open set containing the origin and a locally bounded, continuous viscosity solution of (4.5) in the open set such that $V(0)=0$ and $V(x) \rightarrow+\infty$ for $x \rightarrow \partial \tilde{\mathcal{O}}$.

Proof. We prove only (i), since the proof of assertion (ii) is similar. Note that by Remark 4.3 the functions $v$ and $V$ can be taken to be defined through (4.4) and the running cost $\tilde{g}$. In the following we work with this representation. Again by $\tilde{\varphi}(t, x, u)$ we denote the solutions of (4.4).

Claim 1: If $w^{-}$is a bounded continuous subsolution of (4.6) on $\mathbb{R}^{n}$ with $w^{-}(0) \leq 0$, then $w^{-} \leq v$.

By the upper semicontinuity of $w^{-}$and $w^{-}(0) \leq 0$ we obtain that for every $\varepsilon>0$ there exists a $\delta>0$ with $w^{-}(x) \leq \varepsilon$ for all $x \in \mathbb{R}^{n}$ with $\|x\| \leq \delta$. Now we distinguish two cases:

(i) $x_{0} \in \mathcal{D}_{0}$ : We choose $u^{*} \in \mathcal{U}$ such that $v\left(x_{0}\right)+\varepsilon>\tilde{J}\left(x_{0}, u^{*}\right)=1-\tilde{G}\left(x_{0}, \infty, u^{*}\right)$. In particular, using (H3) and the final statement of Remark 4.3 this implies that there exists a sequence $t_{k} \rightarrow \infty$ such that $\tilde{\varphi}\left(t_{k}, x_{0}, u^{*}\right) \rightarrow 0$ as $k \rightarrow \infty$. Thus it follows from the lower optimality principle (4.7) and the definition of $v$ that

$$
\begin{aligned}
w^{-}\left(x_{0}\right) & \leq \limsup _{k \rightarrow \infty} 1+\tilde{G}\left(x_{0}, t_{k}, u^{*}\right)\left(w^{-}\left(\tilde{\varphi}\left(t_{k}, x_{0}, u^{*}\right)\right)-1\right) \\
& \leq 1+\tilde{G}\left(x_{0}, \infty, u^{*}\right)(\varepsilon-1) \leq v\left(x_{0}\right)+2 \varepsilon
\end{aligned}
$$

which shows the claim as $\varepsilon>0$ was arbitrary.

(ii) $x_{0} \notin \mathcal{D}_{0}$ : In this case by Proposition 3.3 it is sufficient to show that $w^{-}\left(x_{0}\right) \leq 1$. Let $M$ be a bound on $\left|w^{-}\right|$.

In the following we use $t$ as the variable for (2.1) and $\tau$ for the time-variable of (4.4) as in Remark 4.1. Since $\tilde{\varphi}\left(\tau, x_{0}, u\right) \notin B(0, r)$ for all $\tilde{u} \in \mathcal{U}$ and all $\tau \geq 0$ by (H3) and Remark 4.3 we have

$$
\int_{0}^{\tau} \tilde{g}\left(\tilde{\varphi}\left(s, x_{0}, u\right), \tilde{u}(s)\right) d s=\int_{0}^{t(\tau)} g\left(\phi\left(s, x_{0}, u\right) d s \geq g_{r} t(\tau)\right.
$$

for the constant $g_{r}>0$ from (H3). Therefore $\tilde{G}\left(x_{0}, \tau, \tilde{u}\right) \leq \exp \left(-\tilde{g}_{r} t(\tau)\right)$ for all $\tau \geq 0, \tilde{u} \in \mathcal{U}$. Hence

$$
1+\tilde{G}\left(x_{0}, \tau, \tilde{u}\right)\left(w^{-}\left(\tilde{\varphi}\left(\tau, x_{0}, \tilde{u}\right)\right)-1\right) \leq 1+\exp \left(-g_{r} t(\tau)\right)(M+1)
$$

for all $\tilde{u} \in \mathcal{U}$ and the result follows by (4.7) as the right hand side tends to 1 for $\tau \rightarrow \infty$.

Therefore Claim 1 is proved. To conclude the proof we now consider 
Claim 2: Let $w^{+}$be a bounded continuous supersolution of (4.2) on $\mathbb{R}^{n}$ with $w^{+}(0) \geq 0$. Then $w^{+} \geq v$.

Again we distinguish two cases.

(i) $x_{0} \notin \mathcal{D}_{0}$ : In this case we know $v\left(x_{0}\right)=1$ and it is sufficient to show $w^{+}\left(x_{0}\right) \geq 1$. In order to prove this inequality by contradiction, we assume $w^{+}\left(x_{0}\right)=1-\delta$ for some suitable $\delta>0$ and pick the set $\Omega=\left\{x \in \mathbb{R}^{n} \mid w^{+}(x)<1-\delta / 2\right\}$. Since $x_{0} \notin \mathcal{D}_{0}$, for all $\tilde{u} \in \mathcal{U}$ we have the inequality $\tilde{g}\left(t, x_{0}, \tilde{u}\right) \geq g_{r}$ for all $t \geq 0$. Let $M>0$ be a bound on $\left|w^{+}\right|$. Using (4.8) this implies

$$
\begin{aligned}
w^{+}\left(x_{0}\right) & \geq \sup _{\tau \in\left[0, T_{e x}\left(x_{0}, \tilde{u}, \Omega\right)\right]}\left\{1+\tilde{G}\left(x_{0}, \tau, \tilde{u}\right)\left(w^{+}\left(\tilde{\varphi}\left(\tau, x_{0}, \tilde{u}\right)\right)-1\right)\right\} \\
& \geq \sup _{\tau \in\left[0, T_{e x}\left(x_{0}, \tilde{u}, \Omega\right)\right]}\left\{\left[1-\exp \left(-\tau g_{r}\right)\right]-\exp \left(-\tau g_{r}\right) M\right\} .
\end{aligned}
$$

If $T_{e x}\left(x_{0}, \tilde{u}, \Omega\right)=\infty$ then this expression equals 1 , hence we obtain obtain $w^{+}\left(x_{0}\right) \geq$ 1 which contradicts our assumption $w^{+}\left(x_{0}\right)=1-\delta<1$. If $T_{e x}\left(x_{0}, \tilde{u}, \Omega\right)$ is finite then we obtain

$$
\begin{aligned}
w^{+}\left(x_{0}\right) & \geq \sup _{\tau \in\left[0, T_{e x}\left(x_{0}, \tilde{u}, \Omega\right)\right]}\left\{1+\tilde{G}\left(x_{0}, \tau, \tilde{u}\right)\left(w^{+}\left(\tilde{\varphi}\left(\tau, x_{0}, \tilde{u}\right)\right)-1\right)\right\} \\
& \geq 1+\tilde{G}\left(x_{0}, T_{e x}\left(x_{0}, \tilde{u}, \Omega\right), \tilde{u}\right)(1-\delta / 2-1) \geq 1-\delta / 2
\end{aligned}
$$

(observing that $\tilde{G}\left(x_{0}, t, \tilde{u}\right) \leq 1$ ) which again contradicts our assumption $w^{+}\left(x_{0}\right)=$ $1-\delta$.

(ii) $x_{0} \in \mathcal{D}_{0}$ : In this case we know that $v\left(x_{0}\right)<1$, hence for $w^{+}\left(x_{0}\right) \geq 1$ there is nothing to show. Thus we can assume $w^{+}\left(x_{0}\right)=1-\delta$ for some suitable $\delta>0$ and again consider the set $\Omega=\left\{x \in \mathbb{R}^{n} \mid w^{+}(x)<1-\delta / 2\right\}$. Now fix $\varepsilon>0$ with $\varepsilon<\delta / 2$ implying

$$
w^{+}\left(x_{0}\right)+\varepsilon<1-\delta / 2 .
$$

Then (4.8) yields the existence of a control function $u_{\varepsilon} \in \mathcal{U}$ with

$$
w^{+}\left(x_{0}\right)+\varepsilon \geq \sup _{t \in\left[0, T_{e x}\left(x_{0}, u_{\varepsilon}, \Omega\right)\right]}\left\{1+\tilde{G}\left(x_{0}, t, u_{\varepsilon}\right)\left(w^{+}\left(\tilde{\varphi}\left(t, x_{0}, u_{\varepsilon}\right)\right)-1\right)\right\} .
$$

If $T_{e x}\left(x_{0}, u_{\varepsilon}, \Omega\right)<\infty$ then (4.9), (4.10) and $\tilde{G}\left(x_{0}, t, u_{\varepsilon}\right) \leq 1$ imply

$$
1-\delta / 2>w^{+}\left(x_{0}\right)+\varepsilon \geq 1+\tilde{G}\left(x_{0}, t, u_{\varepsilon}\right)(1-\delta / 2-1) \geq 1-\delta / 2,
$$

i.e., a contradiction. Thus we obtain $T_{e x}\left(x_{0}, u_{\varepsilon}, \Omega\right)=\infty$.

Now for each $\eta>0$ we find $t$ such that $\left\|\tilde{\varphi}\left(t, x_{0}, u_{\varepsilon}\right)\right\| \leq \eta$, because otherwise - as in the first inequality of case (i), above - the right hand side in (4.10) would be equal to 1 contradicting (4.9). The continuity of $w^{+}$and the assumption $w^{+}(0) \geq 0$ imply that there exists a $\eta_{1}>0$ such that

$$
w^{+}(x) \geq-\varepsilon \text { for all }\|x\| \leq \eta_{1} .
$$

On the other hand, since $v(0)=0$ and $v$ is continuous we find $\eta_{2}>0$ such that

$$
v(x) \leq \varepsilon \text { for all }\|x\| \leq \eta_{2} .
$$

Combining these results, we can conclude that for all sufficiently large times $t>0$ we have

$$
\tilde{w}^{+}\left(\tilde{\varphi}\left(t, x_{0}, u_{\varepsilon}\right)\right) \geq v\left(\tilde{\varphi}\left(t, x_{0}, u_{\varepsilon}\right)\right)-2 \varepsilon .
$$


Thus using (4.10), (3.5) and the inequality $\tilde{G}\left(x_{0}, t_{n}, u_{n}\right) \leq 1$ for sufficiently large $t>0$ we can conclude

$$
\begin{aligned}
w^{+}\left(x_{0}\right) & \left.\geq 1+\tilde{G}\left(x_{0}, t, u_{\varepsilon}\right)\left(w^{+}\left(\tilde{\varphi}\left(t, x_{0}, u_{\varepsilon}\right)\right)-1\right)\right\}-\varepsilon \\
& \left.\geq 1+\tilde{G}\left(x_{0}, t, u_{\varepsilon}\right)\left(v\left(\tilde{\varphi}\left(t, x_{0}, u_{\varepsilon}\right)\right)-1\right)\right\}-3 \varepsilon \\
& \geq v\left(x_{0}\right)-3 \varepsilon
\end{aligned}
$$

which shows Claim 2, as $\varepsilon>0$ is arbitrary.

Finally, since every viscosity solution $\tilde{w}$ is both sub- and supersolution, the combination of Claim 1 and 2 proves the lemma.

Proof. of Theorem 4.4 All properties follow from the fact that by Lemma 4.9 the functions $V$ and $v$ defined by (3.2) and (3.3) are the unique continuous viscosity solutions for (4.6) and (4.5), respectively.

(i) and (ii): By Corollary 4.6 all viscosity solutions to (4.6) and (4.5) equations are also viscosity solutions of (4.2) and (4.1), respectively, and vice versa. Hence, also the viscosity solutions of (4.2) and (4.1) must be unique.

(iii): By Proposition 3.3 applied to $\tilde{f}$ and $\tilde{g}$ we obtain that $v=\tilde{v}$ and $V=\tilde{V}$ characterize $\mathcal{D}_{0}$ for $\tilde{f}$. Now every trajectory of $f$ is also a trajectory of $\tilde{f}$ for a suitably rescaled control function, and vice versa. Hence the respective domains of null controllability coincide, thus $v$ and $V$ characterize this set.

(iv): The stated behavior at the boundary of $\mathcal{D}_{0}$ follows from Proposition 3.6, since $\tilde{f}$ satisfies (H4b) because it is globally bounded. The behavior for $\left\|x_{k}\right\| \rightarrow \infty$ follows from Remark 3.2, again since $\tilde{f}$ is bounded.

\section{Approximation with bounded control values}

In this section we consider the bounded approximations $U_{k}=U \cap \operatorname{cl} B(0, k)$ of the (possibly) unbounded set $U$ of control values and the corresponding set $\mathcal{U}_{k}:=L^{\infty}\left([0, \infty), U_{k}\right)$ of control functions. Throughout this section we assume that (H0)-(H2) holds which implies that we can find $g$ meeting (H3)-(H6).

Proposition 5.1. Consider the functions

$$
V_{k}(x)=\inf _{u \in \mathcal{U}_{k}} J(x, u) \quad \text { and } \quad v_{k}(x)=1-e^{V_{k}(x)} .
$$

Then the relations

$$
V(x)=\inf _{k \in \mathbb{N}} V_{k}(x) \quad \text { and } \quad v(x)=\inf _{k \in \mathbb{N}} v_{k}(x)
$$

hold.

Proof. Since $\mathcal{U}_{k} \subseteq \mathcal{U}$ we obviously have the inequality $V_{k}(x) \geq V(x)$. Now let $x \in \mathcal{D}_{0}$ and $u \in \mathcal{U}$ be such that

$$
J(x, u) \leq V(x)+\varepsilon
$$

for some $\varepsilon>0$. Since $u \in \mathcal{U}$ there exists $k_{0} \in \mathbb{N}$ such that $\|u\|_{\infty} \leq k_{0}$, hence $u \in \mathcal{U}_{k_{0}}$. This implies

$$
\inf _{k \in \mathbb{N}} V_{k}(x) \leq V_{k_{0}}(x) \leq V(x)+\varepsilon .
$$

Since $\varepsilon$ was arbitrary this shows the claim on $\mathcal{D}_{0}$, both for $V$ and $v$. For $x \notin \mathcal{D}_{0}$ we have $V_{k}(x)=V(x)=\infty$ and $v_{k}(x)=v(x)=1$ which shows the claim also in this case. 
Remark 5.2. If the assumptions of Proposition 3.6 hold, then, since $v_{k}$ is decreasing in $k$, Dini's Theorem yields that $v_{k}$ converges to $v$ locally uniformly on $\mathbb{R}^{n}$.

For the following proposition recall the definition of set limits, which for a sequence of sets $X_{k}$ are given by

$$
\limsup _{k \rightarrow \infty} X_{k}:=\bigcap_{k \in \mathbb{N}} \bigcup_{m \geq k} X_{m} \text { and } \liminf _{k \rightarrow \infty} X_{k}:=\bigcup_{k \in \mathbb{N}} \bigcap_{m \geq k} X_{m}
$$

and, if these two sets coincide,

$$
\lim _{k \rightarrow \infty} X_{k}:=\limsup _{k \rightarrow \infty} X_{k}=\liminf _{k \rightarrow \infty} X_{k} .
$$

Proposition 5.3. Consider the sets

$$
\mathcal{D}_{k}:=\left\{x \in \mathbb{R}^{n} \mid \text { there exists } u \in \mathcal{U}_{k} \text { with }\|\varphi(t, x, u)\| \rightarrow 0 \text { for } t \rightarrow \infty\right\} .
$$

Then the set limit $\lim _{k \rightarrow \infty} \mathcal{D}_{k}$ exists and satisfies

$$
\mathcal{D}_{0}=\lim _{k \rightarrow \infty} \mathcal{D}_{k} .
$$

Proof. Since we have that $V \leq \ldots \leq V_{k+1} \leq V_{k}$ we obtain the inclusion

$$
\mathcal{D}_{k} \subseteq \mathcal{D}_{k+1} \subseteq \ldots \subseteq \mathcal{D}_{0} .
$$

It follows that $\bigcup_{m \geq k} \mathcal{D}_{m} \subseteq \mathcal{D}_{0}$ for each $k$ and hence

$$
\limsup _{k \rightarrow \infty} \mathcal{D}_{k}=\bigcap_{k \in \mathbb{N}} \bigcup_{m \geq k} \mathcal{D}_{m} \subseteq \mathcal{D}_{0} .
$$

On the other hand, if $x \in \mathcal{D}_{0}$ then for any $\varepsilon>0$ there exists $k_{0} \in \mathbb{N}$ with $V_{k}(x) \leq$ $V(x)+\varepsilon$ for all $k \geq k_{0}$. This implies that $x \in \mathcal{D}_{k}$ for all $k \geq k_{0}$ and consequently $x \in \bigcap_{m \geq k_{0}} \mathcal{D}_{m}$. This implies

$$
x \in \bigcup_{k \in \mathbb{N}} \bigcap_{m \geq k} \mathcal{D}_{m}=\liminf _{k \rightarrow \infty} \mathcal{D}_{k},
$$

and since $x \in \mathcal{D}_{0}$ was arbitrary we obtain

$$
\mathcal{D}_{0} \subseteq \liminf _{k \rightarrow \infty} \mathcal{D}_{k},
$$

which shows the claim.

Remark 5.4. This Proposition implies that for any compact set $K \subset \mathbb{R}^{n}$ the convergence

$$
d_{H}\left(K \cap \mathcal{D}_{k}, K \cap \mathcal{D}_{0}\right) \rightarrow 0
$$

in the Hausdorff metric holds (see e.g. [3, Proposition 1.1.5]). In particular, if $\mathcal{D}_{0}$ is bounded then we obtain uniform convergence of $\mathcal{D}_{k}$ to $\mathcal{D}_{0}$ in the Hausdorff metric.

In particular, this implies that for any compact set $K \subset \mathcal{D}_{0}$ we obtain $K \subset \mathcal{D}_{k}$ for all sufficiently large $k$. Thus, in order to steer the system to 0 from a compact subset $K \subset \mathcal{D}_{0}$ it is sufficient to consider bounded control functions. 


\section{ExAmples}

In this section we discuss the necessity of some of our assumptions. Also it is explained how the classical case of linear quadratic control fits within the present framework.

Example 6.1. Consider the one dimensional dynamics

$$
\dot{x}(t)=(x(t)-1)(u(t)+1)+1=x(t)(u(t)+1)-u(t), \quad t \geq 0,
$$

where $U=\mathbb{R}$. The origin is an equilibrium point so that (H1) is satisfied, while $x=1$ is repulsive, in the sense that any trajectory starting from $x_{0} \geq 1$ cannot reach the origin. With this it is easy to see that $\mathcal{D}_{0}=(-\infty, 1)$. Furthermore, (H0) is satisfied with $\gamma(u)=|u|$.

Now consider the cost function $g_{1}(x, u)=|x|$, which satisfies (H3) and (H4) but neither (H5) nor $(\mathrm{H} 6)$. For $x_{0} \in(0,1)$ and an arbitrary constant $\alpha>0$ choose

$$
u(t)=\frac{-\alpha-1}{\phi(t)-1} \chi_{\left[0, x_{0} / \alpha\right]}(t)
$$

where $\chi_{\left[0, x_{0} / \alpha\right]}$ denotes the indicator function of the interval $\left[0, x_{0} / \alpha\right]$. The corresponding solution of (6.1) is given by

$$
\phi(t)=\left(x_{0}-\alpha t\right) \chi_{\left[0, x_{0} / \alpha\right]}(t),
$$

Observe that for $x_{0}$ close to 1 we need a very large control to start to move towards the origin. This is because the control $u$ is multiplied by $x-1$.

Calculating the corresponding cost we obtain

$$
V_{1}\left(x_{0}\right) \leq \int_{0}^{\infty} g_{1}(\phi(t), u(t)) d t=x_{0}^{2} / 2 \alpha
$$

and therefore sending $\alpha \rightarrow+\infty$, it follows that $V_{1}\left(x_{0}\right)=0$ for any $x_{0} \in(0,1)$. Of course, $V_{1}(x)=\infty$ for $x \geq 1$. Summarizing this shows that $v_{1}$ is discontinuous on $\mathbb{R}$ and not a control Lyapunov function on $\mathcal{D}_{0}$.

On the other hand setting $g_{2}(x, u)=|x|+|u|$ a cost function satisfying (H6) is obtained. To analyze the associated value functions fix $x_{0} \in(0,1)$ and choose a control $u$ such that $\phi(t):=\phi(t, x, u) \rightarrow 0$. We will assume that $\phi$ is strictly decreasing as otherwise it is clearly not optimal. Now let $T>0$ be a time such that $\phi(T)>0$, then we have

$$
\begin{aligned}
J_{2}(x, u) \geq & \int_{0}^{T} \phi(t)+u(t) d t=\int_{0}^{T} \phi(t)+\frac{\phi(t)-\dot{\phi}(t)}{1-\phi(t)} d t \geq \\
& \int_{0}^{T} \frac{-\dot{\phi}(t)}{1-\phi(t)} d t=\log (1-\phi(T))-\log \left(1-x_{0}\right) .
\end{aligned}
$$

As $\phi(T)$ approaches 0 (in finite or infinite time) this calculation shows that $V_{2}\left(x_{0}\right) \geq$ $-\log \left(1-x_{0}\right)$ for $x_{0} \in(0,1)$ so that in particular $v_{2}$ is continuous on $\mathbb{R}$ and a control Lyapunov function on $\mathcal{D}_{0}$ (where we leave the assertion for $(-\infty, 0)$ to the reader).

Finally note that a combination of the previous examples leads to an intermediate situation. To this end let $h: \mathbb{R} \rightarrow[0,1]$ be a continuous function such that $h(x)=1$ if $x \in(-\infty, 1 / 2], h(x)=0$ for $x \in[3 / 4, \infty)$ and let $g_{3}(x, u)=|x|+h(x)|u|$. Then it follows for $x \in[0,1 / 2]$ that $V_{3}(x)=V_{2}(x) \geq-\log (1-x)$ by the considerations on $g_{2}$, whereas for $x \in(3 / 4,1)$ we have $V_{3}(x)=V(3 / 4)$ using that $V_{1}$ is constant on that interval. In this example (H5) and (H6) are not satisified, $v$ is not continuous and $V$ is a control Lyapunov function only on a subset of $\mathcal{D}_{0}$. 
Example 6.2. Finally we show that the classical linear quadratic control problem fits into our setup. This problem is obtained if we set

$$
f(x, u)=A x+B u \text { and } g(x, u)=x^{T} Q x+u^{T} R u,
$$

where $A, B, Q, R$ are matrices of appropriate dimensions with $Q$ and $R$ being symmetric and positive definite.

By direct computations one sees that these functions satisfiy (H0) for any $\gamma \in$ $\mathcal{K}_{\infty},(\mathrm{H} 1),(\mathrm{H} 3)$ and (H5). The linear system also satisfies (H2'), because it is known that local asymptotic controllability implies the existence of a feedback matrix $F$ such that $A+B F$ is exponentially stable, i.e., this matrix has all its eigenvalues in the open left half plane, which yields (H2') with $\beta(r, t)=K e^{-\lambda t} r$ for suitable constants $K, \lambda>0$. Hence we obtain $\beta(r, t)=\alpha_{2}\left(\alpha_{1}(r) e^{-t}\right)$ with $\alpha_{2}(r)=r^{\lambda}$ which implies (H4') for our $g$ with $\delta=2 / \lambda$ and $C=\|Q+R\|$. Finally, (H6) is satisfied because $g$ grows quadratically in $u$ while $f$ only grows linearly in $u$. Thus, the classical linear quadratic problem is a special case of our setup and the resulting equation (4.1) is given by

$$
\sup _{u \in U}\left\{-D V(x)(A x+B u)-x^{T} Q x-u^{T} R u\right\}=0 .
$$

For the quadratic ansatz $V(x)=x^{T} P x$ with symmetric matrix $P$ we obtain

$$
D V(x)(A x+B u)=x^{T} P(A x+B u)+(A x+B u)^{T} P x .
$$

Assuming $U=\mathbb{R}^{m}$ we can explicitly solve the maximization problem over $u$ by setting the first derivative of the resulting expression to 0 and obtain

$$
u(x)=-R^{-1} B^{T} P x .
$$

Plugging this into (6.3) and multiplying by -1 yields

$$
x^{T} P B R^{-1} B^{T} P x-x^{T} P A x-x^{T} A^{T} P x-x^{T} Q x=0
$$

which is equivalent to

$$
P B R^{-1} B^{T} P-P A-A^{T} P-Q=0,
$$

i.e., (4.1) reduces to the well known algebraic Riccati equation from linear optimal control, see [31, Section 8.4].

\section{REFERENCES}

[1] Z. ARTstein, Stabilization with relaxed controls, Nonlinear Anal., 7 (1983), pp. 1163-1173.

[2] J.-P. Aubin And A. Cellina, Differential inclusions, vol. 264 of Grundlehren der Mathematischen Wissenschaften, Springer-Verlag, Berlin, 1984.

[3] J.-P. Aubin And H. Frankowska, Set-valued analysis, vol. 2 of Systems \& Control: Foundations \& Applications, Birkhäuser Boston Inc., Boston, MA, 1990.

[4] M. BARd AND I. CAPUZzo-Dolcetta, Optimal control and viscosity solutions of HamiltonJacobi-Bellman equations, Systems \& Control: Foundations \& Applications, Birkhäuser, Boston, MA, 1997.

[5] M. BARDi AND F. DA LIO, On the Bellman equation for some unbounded control problems, NoDEA Nonlinear Differential Equations Appl., 4 (1997), pp. 491-510.

[6] G. BArles, Solutions de viscosité des équations de Hamilton-Jacobi, vol. 17 of Mathématiques \& Applications (Berlin) [Mathematics \& Applications], Springer-Verlag, Paris, 1994.

[7] R. W. Brockett, Asymptotic stability and feedback stabilization, in Differential geometric control theory (Houghton, Mich., 1982), vol. 27 of Progr. Math., Birkhäuser Boston, Boston, MA, 1983, pp. 181-191. 
[8] F. Camilli, L. Grüne, and F. Wirth, A generalization of Zubov's method to perturbed systems, SIAM J. Control Optim., 40 (2001), pp. 496-515.

[9] F. H. Clarke, Y. S. Ledyaev, L. Rifford, and R. J. Stern, Feedback stabilization and Lyapunov functions, SIAM J. Control Optim., 39 (2000), pp. 25-48 (electronic).

[10] F. H. Clarke, Y. S. Ledyaev, E. D. Sontag, and A. I. Subbotin, Asymptotic controllability implies feedback stabilization, IEEE Trans. Automat. Control, 42 (1997), pp. 1394-1407.

[11] F. H. Clarke, Y. S. Ledyaev, and R. J. Stern, Asymptotic stability and smooth Lyapunov functions, J. Differential Equations, 149 (1998), pp. 69-114.

[12] J.-M. Conon, A necessary condition for feedback stabilization, Systems Control Lett., 14 (1990), pp. 227-232.

[13] F. DA LIO, On the Bellman equation for infinite horizon problems with unbounded cost functional, Appl. Math. Optim., 41 (2000), pp. 171-197.

[14] S. Dubljevič And N. KazAntsis, A new Lyapunov design approach for nonlinear systems based on Zubov's method, Automatica, 38 (2002), pp. 1999-2007.

[15] R. A. Freeman and P. V. Kokotovic, Inverse optimality in robust stabilization, SIAM J. Control Optim., 34 (1996), pp. 1365-1391.

[16] R. A. Freeman and P. V. Kokotović, Robust nonlinear control design, Systems \& Control: Foundations \& Applications, Birkhäuser Boston Inc., Boston, MA, 1996. State-space and Lyapunov techniques.

[17] P. GiesL, Eine Charakterisierung der Einzugsbereiche von Gleichgewichtspunkten und periodischen Orbits dynamischer Systeme, $\mathrm{PhD}$ thesis, Technische Universität München, Germany, 2000.

[18] S. F. HAfstein, A constructive converse Lyapunov theorem on exponential stability, Discrete Contin. Dyn. Syst., 10 (2004), pp. 657-678.

[19] W. Hahn, Stability of motion, Translated from the German manuscript by Arne P. Baartz. Die Grundlehren der mathematischen Wissenschaften, Band 138, Springer-Verlag New York, Inc., New York, 1967.

[20] Y. KuRCVEIL', On the inversion of the second theorem of Lyapunov on stability of motion, Czechoslovak Math. J., 6(81) (1956), pp. 217-259, 455-484.

[21] Y. S. Ledyaev and E. D. Sontag, A Lyapunov characterization of robust stabilization, Nonlinear Anal., 37 (1999), pp. 813-840.

[22] Y. Lin, E. D. Sontag, AND Y. WANG, A smooth converse Lyapunov theorem for robust stability, SIAM J. Control Optim., 34 (1996), pp. 124-160.

[23] M. Malisoff, Further results on Lyapunov functions and domains of attraction for perturbed asymptotically stable systems. to appear in Dynam. Contin. Discrete Impuls. Systems, 2004.

[24] J. L. MASSERA, On Liapunov's condition of stability, Annals of Math., 50 (1949), pp. 705721.

[25] L. RIFForD, Existence of Lipschitz and semiconcave control-Lyapunov functions, SIAM J. Control Optim., 39 (2000), pp. 1043-1064 (electronic).

[26] - Semiconcave control-Lyapunov functions and stabilizing feedbacks, SIAM J. Control Optim., 41 (2002), pp. 659-681 (electronic).

[27] E. P. RYAN, On Brockett's condition for smooth stabilizability and its necessity in a context of nonsmooth feedback, SIAM J. Control Optim., 32 (1994), pp. 1597-1604.

[28] R. Sepulchre, M. Janković, and P. V. Kokotović, Constructive nonlinear control, Communications and Control Engineering Series, Springer-Verlag, Berlin, 1997.

[29] E. D. SontaG, A Lyapunov-like characterization of asymptotic controllability, SIAM J. Control Optim., 21 (1983), pp. 462-471.

[30] — Comments on integral variants of ISS, Systems Control Lett., 34 (1998), pp. 93-100.

[31] E. D. Sontag, Mathematical Control Theory: Deterministic Finite Dimensional Systems, no. 6 in Texts in Applied Mathematics, Springer-Verlag, New York, 2nd ed., 1998.

[32] E. D. SontaG, Stability and stabilization: discontinuities and the effect of disturbances, in Nonlinear analysis, differential equations and control (Montreal, QC, 1998), vol. 528 of NATO Sci. Ser. C Math. Phys. Sci., Kluwer Acad. Publ., Dordrecht, 1999, pp. 551-598.

[33] P. SoRAVIA, Optimality principles and representation formulas for viscosity solutions of Hamilton-Jacobi equations. I. Equations of unbounded and degenerate control problems without uniqueness, Adv. Differential Equations, 4 (1999), pp. 275-296. 
[34] - Optimality principles and representation formulas for viscosity solutions of HamiltonJacobi equations. II. Equations of control problems with state constraints, Differential Integral Equations, 12 (1999), pp. 275-293.

[35] A. R. Teel and L. Praly, A smooth Lyapunov function from a class- $\mathcal{K} \mathcal{L}$ estimate involving two positive semidefinite functions, ESAIM Control Optim. Calc. Var., 5 (2000), pp. 313-367.

[36] F. W. Wilson, JR., The structure of the level surfaces of a Lyapunov function, J. Differential Equations, 3 (1967), pp. 323-329.

[37] V. I. Zubov, Methods of A. M. Lyapunov and their application, P. Noordhoff Ltd, Groningen, 1964.

Sez. di Matematica per l'Ingegneria, Dip. di Matematica Pura e Applicata, Università de l'Aquila, 67040 Roio Poggio (AQ), Italy

E-mail address: camilli@ing.univaq.it

Mathematisches Institut, Fakultät für Mathematik und Physik, Universität Bayreuth, 95440 Bayreuth, Germany

E-mail address: lars.gruene@uni-bayreuth.de

The Hamilton Institute, NUi Maynooth, Maynooth, Co. Kildare, Ireland

E-mail address: fabian.wirth@may.ie 